\title{
Lessons of the month 1: Longitudinal extensive transverse myelitis following AstraZeneca COVID-19 vaccination
}

\author{
Authors: Alp AA Notghi, ${ }^{A}$ Joseph Atley $^{B}$ and Mark Silva ${ }^{C}$
}

\begin{abstract}
Longitudinal extensive transverse myelitis (LETM) is a rare but recognised complication of vaccination. We report the case of a 58-year-old man admitted to hospital 10 days after his first AstraZeneca COVID-19 vaccination with progressive neurological symptoms and signs, and investigations and imaging consistent with LETM.

This case reviews the literature and the investigative process behind excluding other diagnoses given the patient's background of pulmonary sarcoidosis. It is unique in being the first UK report of a case of LETM with a strong temporal link to COVID-19 vaccination.
\end{abstract}

KEYWORDS: COVID-19, vaccination, myelitis, AstraZeneca, longitudinal extensive transverse myelitis

DOI: $10.7861 /$ clinmed.2021-0470

\section{Case presentation}

A 58-year-old White man was admitted urgently with progressive numbness in his lower limbs over 3 days, allodynia up to chest level, genital dysaesthesia and an episode of urinary incontinence. On examination, there was hyperaesthesia below $\mathrm{T} 7$, hyperreflexia in all four limbs (including ankle clonus) but with preserved motor power, post-void urinary retention and normal cranial nerves.

Following his first dose of the AstraZeneca COVID-19 vaccine, he had experienced transitory flu-like symptoms and injection site pain which resolved within 24 hours. His neurological symptoms started 7 days after administration of the vaccine. His antecedents included type 2 diabetes mellitus and pulmonary sarcoidosis diagnosed at the age of 32 years. He was taking metformin for his diabetes mellitus, which he had been on for many years, and was on no current treatment for pulmonary sarcoidosis.

Initial investigations showed normal urea and electrolytes, full blood count and C-reactive protein. Magnetic resonance imaging

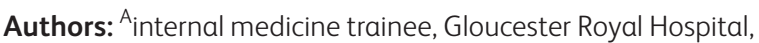
Gloucester, UK; ${ }^{B}$ foundation year-1 doctor, Gloucester Royal Hospital, Gloucester, UK; ' Consultant neurologist, Gloucester Royal Hospital, Gloucester, UK
(MRI) of the whole spine performed to look for compressive lesions at lumbar or thoracic level, or myelopathic features at cervical level, showed abnormal cord signal with T2-weighted and shorttau inversion recovery (STIR) sequence from the thoracic vertebrae levels 2 to 10 (Fig 1). An impression of prominent flow voids in the spinal canal at T9 level raised suspicion of a dural arteriovenous fistulae (DAVF). Further imaging with gadolinium contrast revealed two separate foci of enhancing myelitis, a $20 \mathrm{~mm}$ segment from the T3 vertebral body to the T3/4-disc level and a $14 \mathrm{~mm}$ lesion at the T9/10-disc level (Fig 2).

Over the following week, the patient deteriorated rapidly with progressive dysaesthesia in his hands and fingers, increasing weakness of the lower limbs that made walking impossible, severe allodynia up to neck level and, on examination, absence of vibration sensation below the groin and bilateral upgoing plantar reflexes. The differential diagnosis at this stage included autoimmune longitudinal extensive transverse myelitis (LETM); neurosarcoidosis; HIV or syphilis; deficiencies of B12, folate or copper; neuromyelitis optica; and DAVF. Further MRI of the head and whole spine with contrast revealed a more striking and extensive T2-weighted hyperintense signal abnormality up to C1 level (Fig 3). Repeat images of the thoracic cord suggested flow voids rather than extramedullary serpiginous vessels, and no contrast enhancement of the extra medullary veins pointed away from a diagnosis of DAVF. Cerebrospinal fluid (CSF) analysis revealed a raised protein of $1.68 \mathrm{~g} / \mathrm{L}$, lymphocytic pleocytosis and oligoclonal bands of an identical band pattern to that found in the serum, the latter suggesting a systemic inflammatory response. Additional results from blood and CSF analysis are shown in Table 1.

To investigate the possibility of neurosarcoidosis, computed tomography (CT) of the thorax was performed, which showed calcified mediastinal lymph nodes in keeping with known past sarcoidosis. CT also revealed nodules distributed perilymphatically and within the pulmonary fissures, however, subsequent CT positron emission tomography (CT-PET) showed no evidence of fluorodeoxyglucose uptake within these nodules nor elsewhere to suggest active sarcoidosis. Slit lamp examination and ophthalmoscopy demonstrated no evidence of ocular sarcoidosis, and transthoracic echocardiography was normal.

Treatment was started with intravenous methylprednisolone $1 \mathrm{~g} /$ day for 5 days followed by oral prednisolone at $60 \mathrm{mg} /$ day. As the patient showed no signs of improvement after 10 days on steroids, he was given 5 days of plasma exchange (PLEX). Symptoms improved on the second day of PLEX with improved 
Fig 1. Magnetic resonance imaging (MRI) of the spine showing long segment of abnormal cord signal from the T2 vertebrae to T10. a) Initial noncontrast MRI. b) Short-tau inversion recovery (STIR) MRI showing more pronounced abnormal cord signal.
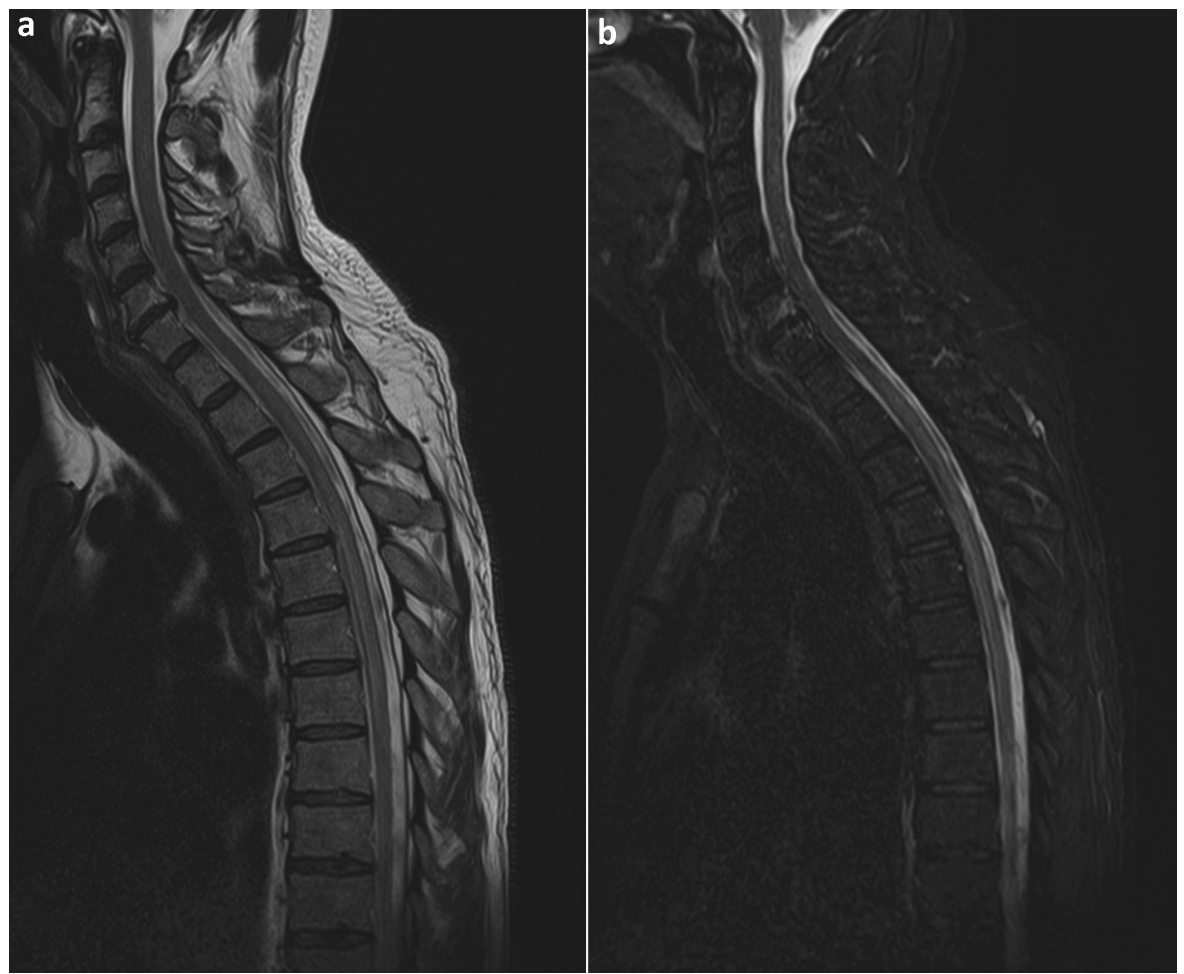

sensation in the hands and he regained an urge to defecate which had previously been lost. The steroid dose was tapered and neurorehabilitation commenced. A repeated MRI of the cervical spine 16 days after commencing PLEX was reassuring, showing patchy and less homogeneous signal change within the cervical cord up to C2 and less oedema of the cord.

Fig 2. Gadolinium-enhanced magnetic resonance imaging. a) $20 \mathrm{~mm}$ focus of enhancing myelitis at the $T 3$ vertebral body (red arrow). b) A second $14 \mathrm{~mm}$ focus of enhancing myelitis at the T9/ 10-disc level (red arrow).

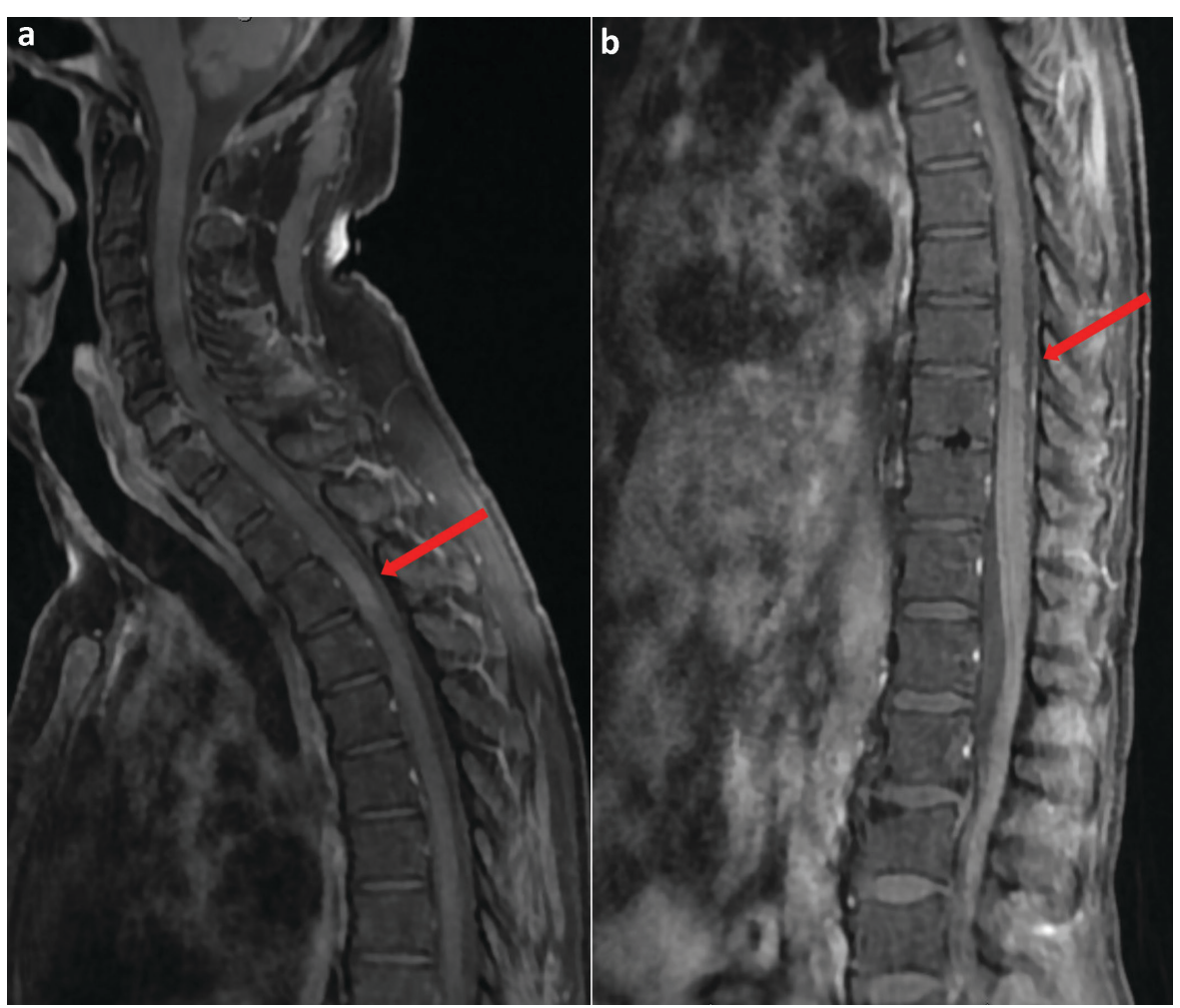


Table 1. Laboratory results

\section{Investigation}

Serum corrected calcium, $\mathrm{mmol} / \mathrm{L}$

C-reactive protein, $\mathrm{mg} / \mathrm{L}$

Plasma glucose (random), $\mathrm{mmol} / \mathrm{L}$

B12, $\mu \mathrm{g} / \mathrm{L}$

Folate, $\mu \mathrm{g} / \mathrm{L}$

Copper, $\mu \mathrm{mol} / \mathrm{L}$

Vitamin 25(OH) D, nmol/L

Hepatitis B surface antibody, mIU/L

Hepatitis B surface antigen

Hepatitis B core antigen

Hepatitis C antibody

Syphilis antibody

HTLV 1 and 2 antibodies

Borrelia burgdorferi immunoglobulin M and $\mathrm{G}$

Human herpes virus 6 PCR

HIV antibody/antigen

Mycoplasma pneumoniae complement fixation test

Mycoplasma agg

Anti-AQP-4 antibody

Anti-Hu antibody

Purkinje cell (anti-Yo) antibody

Anti-Ri antibody

Anti-MOG antibody

CSF protein, $\mathrm{g} / \mathrm{L}$

CSF glucose, $\mathrm{mmol} / \mathrm{L}$

CSF white blood cells, $\times 10^{6} / \mathrm{L}$

CSF red blood cells, $\times 10^{6} / \mathrm{L}$

CSF microscopy and culture

CSF cytology

CSF JC virus PCR

CSF oligoclonal bands

Atypical ANCA

PR3 and MPO specific ANCA

ANA

ENA (Ro, La, Am, RNP, Scl-70 and Jo-1)

Serum ACE, U/L

\section{Result (normal range)}

$2.32(2.1-2.6)$

$2(<10)$

$14(4.1-11)$

$422(180-1,000)$

$9.9(>4.0)$

$14.4(12-25)$

27.1 (deficiency <30)

$<10$

Not detected

Not detected

Not detected

Not detected

Not detected

Not detected

Negative

Not detected

$<16$

Negative

Negative

Negative

Negative

Negative

Negative

$1.62(0.15-0.4)$

7.6

11

40

No organisms seen, no growth

No malignant cells seen

Negative

Oligoclonal bands of identical band pattern to that found in the serum

Positive

Negative

Negative

Negative

$44(8-52)$

\section{Comment}

No significant titres

$100 \%$ lymphocytes

Suggestive of a systemic inflammatory response but not of intrathecal immunoglobulin $\mathrm{G}$ synthesis

Of doubtful significance

$\mathrm{ACE}=$ angiotensin converting enzyme; $\mathrm{ANA}=$ antinuclear antibodies; $\mathrm{ANCA}=$ anti-neutrophil cytoplasmic antibodies; $\mathrm{AQP}-4$ = aquaporin-4; CSF = cerebrospinal fluid; ENA = extractable nuclear antigens; HTLV = human T-lymphotropic virus; $\mathrm{JC}=$ John Cunningham; MOG = myelin oligodendrocyte glycoprotein; MPO = myeloperoxidase; $\mathrm{PCR}=$ polymerase chain reaction. 


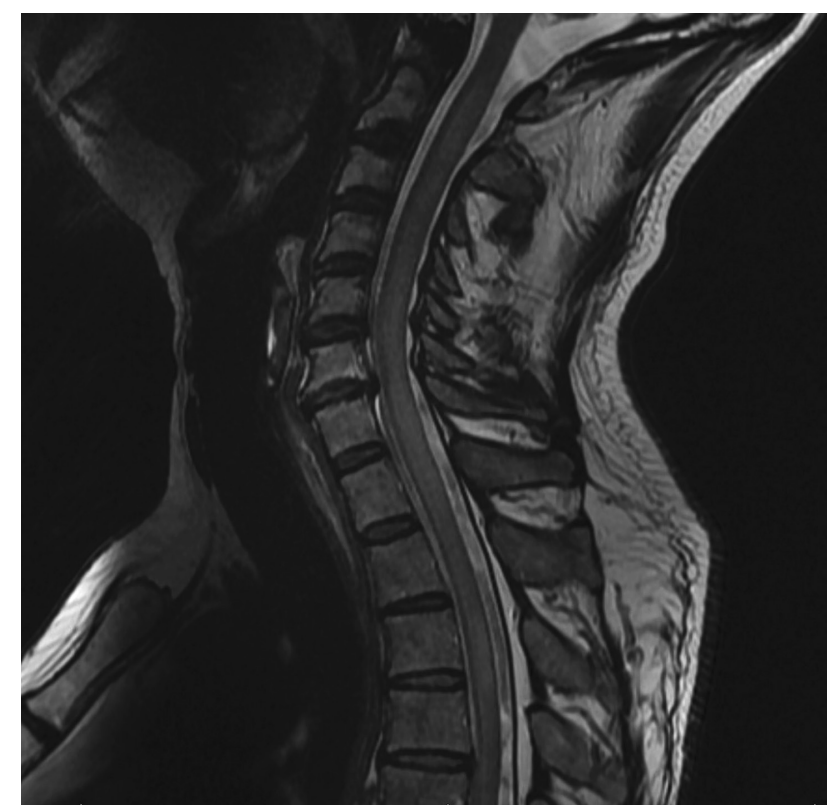

Fig 3. Repeat magnetic resonance imaging (MRI). Progressive changes on T2 weighted sequencing after 4 days when compared with the previous MRI with extensive myelitis.

\section{Discussion}

To identify the cause of this patient's LETM, it was necessary to exclude a range of conditions which might influence long-term management.

Multiple sclerosis was a possibility, however, the absence of past lesions or those disseminated in space on MRI and the absence of evidence for specific intrathecal immunoglobulin $\mathrm{G}$ synthesis on CSF analysis argues against this.

Neuromyelitis optica spectrum disorders may involve a LETM with episodes of optic neuritis. The absence of an optic neuritis in this case along with negative anti-aquaporin- 4 and anti-myelin oligodendrocyte glycoprotein serology argued against this diagnosis. $^{2}$

Neurosarcoidosis can also present as a longitudinal transverse myelitis and was of definite concern given his medical history. ${ }^{3}$ However, a reassuring CT-PET and equivocal response to steroids in contrast to PLEX argues against this aetiology.
Transverse myelitis is a rare condition and it has been linked to a variety of vaccinations as a possible neurological complication. ${ }^{4}$ There have, furthermore, been reports of LETM with a strong temporal link to vaccination. ${ }^{5}$

Trial data looking specifically at the safety and efficacy of the AstraZeneca COVID-19 vaccine reports three possible cases of transverse myelitis during the trial, all latterly thought to be unrelated to the vaccination itself. ${ }^{6}$ This case however demonstrates a strong temporal link with the vaccine after the exclusion of other possible diagnoses through comprehensive investigations.

\section{Conclusion}

This case demonstrates the necessity to consider adverse events from novel vaccines in the context of their rapid development during exceptional times. It additionally demonstrates the diagnostic challenges when faced with such alternative differentials.

\section{References}

1 Thompson AJ, Banwell BL, Barkhof $\mathrm{F}$ et al. Diagnosis of multiple sclerosis: 2017 revisions of the McDonald criteria. Lancet Neurol 2018;17:162-73.

2 Huda S, Whittam D, Bhojak M et al. Neuromyelitis optica spectrum disorders. Clin Med 2019;19:169-76.

3 Fontes-Villalba A, Parratt J. 108 Longitudinally extensive transverse myelitis as the sole manifestation of sarcoidosis. Journal of Neurology, Neurosurgery \& Psychiatry 2018;89:A43.

4 Agmon-Levin N, Kivity S, Szyper-Kravitz M et al. Transverse myelitis and vaccines: a multi-analysis. Lupus 2009;18:1198-204.

5 Akkad W, Salem B, Freeman JW, Huntington MK. Longitudinally extensive transverse myelitis following vaccination with nasal attenuated novel influenza $\mathrm{A}(\mathrm{H} 1 \mathrm{~N} 1)$ vaccine. Arch Neurol 2010;67:1018-20.

6 Voysey M, Clemens SAC, Madhi SA et al. Safety and efficacy of the ChAdOx1 nCoV-19 vaccine (AZD1222) against SARS-CoV-2: an interim analysis of four randomised controlled trials in Brazil, South Africa, and the UK. Lancet 2021;397:99-111.

Address for correspondence: Dr Alp Aslan Andrew Notghi, Department of Neurology, Gloucester Royal Hospital, Great Western Road, Gloucester GL1 3NN, UK.

Email: a.notghi@nhs.net 